\title{
Desindustrialización y crisis en la Barcelona posolímpica: ¿hacia una ciudad dual? ${ }^{1}$
}

Sebastián Sarasa. Universitat Pompeu Fabra, Barcelona, España.

Sergio Porcel. Institut d'Estudis Regionals i Metropolitans de Barcelona, Barcelona, España.

Lara Navarro. Institut d'Estudis Regionals i Metropolitans de Barcelona, Barcelona, España.

Jenniffer Thiers. Universitat de Barcelona, Barcelona, Espańa.

RESUMEN | El tránsito de la sociedad industrial a la posindustrial ha ido acompañado de transformaciones económicas y demográficas con efectos relevantes en la estructura social de los países desarrollados. El aumento de la desigualdad social es uno de tales efectos, manifestado con mayor intensidad en el ámbito urbano, donde también se ha visto afectada la estructura sociorresidencial. Este artículo analiza la evolución de la desigualdad social y de la estructura sociorresidencial en el área metropolitana de Barcelona durante las dos últimas décadas. La hipótesis de partida es que Barcelona, como otras ciudades europeas, no ha tendido en los últimos años hacia un esquema de ciudad dual — caracterizado por la polarización de la estructura social y urbana—, siendo este un modelo más propio de las ciudades estadounidenses. Los resultados confirman esta hipótesis y sitúan a la capital catalana como una ciudad desigual, pero poco segregada.

PALABRAS CLAVE | desigualdad social, transformaciones socioterritoriales, segregación.

ABSTRACT | The transition from industrial to post-industrial society has been accompanied by a set of economic and demographic changes that have triggered significant effects on the social structure of developed countries. The increase in social inequality is one of these effects, especially in urban areas, where socio-residential structure has been affected too. This article analyzes the evolution of social inequality and socio-residential structure in the metropolitan area of Barcelona during the last two decades. The hypothesis is that Barcelona, like others European cities, has not tended in recent years towards a dual city scheme - characterized by the polarization of the social and urban structure-, which fits better with the American cities. The results confirm this hypothesis and place the Catalan capital city as unequal but not too segregated.

KEYWORDS | social inequality, socio-territorial transformations, segregation.

1 Los autores agradecen la financiación obtenida del Programa Recercaixa (Ref. pro8113) y de la Agencia Estatal de Investigació y del Fondo Europeo de Desarrollo Regional (Feder) (Ref cso2016-80484-R). 


\section{Introducción}

El tránsito de la sociedad industrial a la posindustrial ha ido acompañado de un conjunto de transformaciones económicas y demográficas que han desencadenado efectos relevantes en la estructura social de los países desarrollados en el siglo XXI. La innovación tecnológica, el menor poder de los sindicatos y la desregulación de los mercados han alterado la estructura ocupacional y empujan hacia una mayor disparidad salarial (Castells, 1995; Gottschalck \& Smeeding, 1997). Además, la extensión del comercio internacional ha modificado la capacidad protectora de los Estados de Bienestar (Esping-Andersen, 2002; Pierson, 1998; Swank, 2002). El proceso de globalización económica ha comportado, a su vez, un aumento considerable de los movimientos migratorios internacionales, y países que hasta hace relativamente poco tiempo eran emisores de emigrantes han pasado de forma acelerada a ser receptores. La familia, por su parte, también ha evolucionado, reconvirtiéndose en una institución más inestable y de fronteras más difusas, perdiendo así la capacidad protectora que presentaba tiempo atrás ante los riesgos que afrontan los individuos a lo largo del curso vital (Lesthaeghe, 1995). Todo ello ha contribuido, durante las últimas décadas, a un aumento prácticamente generalizado de la desigualdad social, así como al surgimiento de nuevas formas de pobreza (Towsend, 1993).

Los fenómenos mencionados se manifiestan con mayor intensidad en el ámbito urbano, en la medida en que es precisamente en este escenario donde las transformaciones socioeconómicas y demográficas se hacen más extremas. Es en las grandes metrópolis donde se concentran los sectores productivos donde se encuentra una mayor dispersión de ingresos, y es allí donde existe también una mayor proporción de economía informal precaria (Sassen, 1994). Esta repercusión de los cambios económicos estructurales en la desigualdad social urbana ha sido estudiada desde diferentes perspectivas, y los resultados obtenidos dan pie a diversas interpretaciones, no necesariamente contradictorias (Burgers \& Musterd, 2002; Van Kempen, 1994). Los dos enfoques más importantes en esta materia señalan o bien la conformación de una nueva infraclase urbana de marginados y excluidos (Mingione, 1996a; Wacquant, 2001; Wilson, 1987) o bien la existencia de una nueva tendencia hacia la polarización social y socioespacial en las grandes ciudades (Castells \& Mollenkopf, 1991; Harloe \& Fainstein, 1992; Sassen, 1991).

Además de estas transformaciones estructurales de largo recorrido, en la actualidad resulta imposible obviar los efectos coyunturales que está desencadenando la profunda crisis económica que desde 2008 golpea con especial virulencia a los países del sur y del este de Europa. En el contexto espańol, en concreto, la destrucción masiva de ocupación ha desencadenado un aumento generalizado de las situaciones de riesgo de pobreza y de exclusión social, provocado por las situaciones de paro en los hogares, el empeoramiento progresivo de las condiciones laborales y las deficiencias del sistema de protección social (Laparra \& Pérez, 2012). Por lo que respecta a los ámbitos más urbanos, algunos autores ya han apuntado que en Madrid, por ejemplo, la crisis ha castigado de forma más severa a los barrios más desfavorecidos, aumentando considerablemente la fractura social urbana que ya existía antes de la crisis (Méndez \& Prada-Trigo, 2014). En el área metropolitana de Barcelona, 
el impacto social de la crisis ha sido igualmente grave en términos de desigualdad social, aunque todo indica que en la ciudad central la destrucción de ocupación ha sido más moderada que en la primera corona metropolitana (Sarasa, Porcel \& Navarro-Varas, 2013).

Este artículo se orienta a analizar la evolución de la desigualdad social y las dinámicas de segregación residencial socioeconómica en la metrópoli de Barcelona durante las últimas décadas, con el objetivo de contrastar si las tendencias que sigue la capital catalana se acercan o se alejan del modelo de ciudad dual planteado por Saskia Sassen (1991) para las ciudades globales.

\section{Globalización, desindustrialización y ciudad: apuntes del debate teórico}

La influencia que ejercen los cambios económicos estructurales - particularmente el proceso de desindustrialización - respecto a la composición social y la configuración de la estructura sociorresidencial de las grandes ciudades, es un tema que ha sido objeto de estudio en el ámbito de la sociología urbana desde los años ochenta. Uno de los primeros autores que introdujo esta perspectiva fue William J. Wilson (1987), quien vincula el cambio del sistema productivo con la transformación de los guetos afroamericanos. A raíz de este estudio, diversos autores han enfatizado la contribución del nuevo contexto económico a la aparición de enclaves de exclusión social en las grandes ciudades (Massey \& Denton, 1993; Mingione, 1996b). En estos lugares se concentra la población expulsada del mercado laboral urbano, que corre el peligro de cronificar su situación de pobreza y de precariedad ante la falta de oportunidades que le ofrece el nuevo mercado de trabajo, sea por su bajo nivel de formación y su baja cualificación laboral (Gregg \& Wandsworth, 2002), o por las dificultades ańadidas que supone residir en áreas segregadas y degradadas (Atkinson \& Kintrea, 2001; Burgers \& Musterd, 2002; Wacquant, 2007).

Paralelamente a esta línea de estudio focalizada únicamente en la pobreza urbana, Saskia Sassen (1991) introdujo en los ańos noventa una nueva perspectiva de análisis sobre los efectos que están ocasionando los procesos de desindustrialización y de globalización en las grandes ciudades. Su enfoque, más amplio, considera las transformaciones que se producen en el conjunto de la estructura social y urbana de las metrópolis. La autora pone por primera vez de relieve que tres de las ciudades más importantes del mundo -Nueva York, Londres y Tokio- se estaban polarizando, tanto en su composición social como en su estructura sociorresidencial. Según Sassen, el tránsito industrial-posindustrial comporta dos procesos que explican el aumento de la desigualdad social entre la población urbana. Por un lado, la terciarización del sistema productivo; y por otro, el ascenso y la relevancia que adquieren en la nueva estructura del sistema productivo sectores como las finanzas, las telecomunicaciones y las tecnologías informáticas. Esta transformación genera un cambio en la demanda de mano de obra que afecta a la estructura ocupacional, incrementándose sobre todo los trabajadores del sector servicios altamente cualificados, así como los no cualificados que los asisten desempeńando otro tipo de tareas (hostelería, comercios, cuidados, etc.), lo cual se traduce en una desigualdad creciente de los salarios de los trabajadores urbanos (Sassen, 1996). Este fenómeno 
es precisamente el que se recoge en el concepto de ciudad dual, que expresa la tendencia hacia la polarización en diferentes dimensiones de la realidad urbana, como el uso del espacio, el mercado de trabajo, el mercado inmobiliario y la estructura de consumo (Sassen, 1991, 1994).

Pero el planteamiento de Sassen no está exento de críticas y algunos autores han cuestionado la simplicidad del concepto de ciudad dual o su falta de claridad, introduciendo matices y generando un interesante debate en torno a esta cuestión (Burgers \& Musterd, 2002; Castells \& Mollenkopf, 1991; Harloe \& Fainstein, 1992; Reichl, 2007; Van Kempen, 1994). Manuel Castells y John Mollenkopf (1991) son los menos críticos con el modelo teórico de Sassen y consideran que, a pesar de su simplicidad, el concepto de ciudad dual sobre todo es útil a nivel ideológico, en la medida en que consigue alertar de la desigualdad, la explotación y la opresión que tienen lugar en las ciudades contemporáneas, rompiendo de este modo con la idea de la ciudad como comunidad integrada. Por otro lado, Michael Harloe y Susan Fainstein (1992) destacan la potencialidad del concepto de ciudad dual como hipótesis de partida, pero argumentan que la reestructuración económica tiene en la realidad urbana un impacto social y espacial más complejo que el simple contraste entre dos o tres clases sociales. Precisamente Chris Hamnett (1994) profundiza en esta última idea, aludiendo a que en la transformación de la estructura social no solo intervienen la globalización y la desindustrialización, sino que también lo hacen otros factores, como los cambios en la estructura de hogares o los efectos redistributivos del Estado del Bienestar. Por lo tanto, todo indica que la teoría de Sassen no es extrapolable a todas las ciudades occidentales (Hamnett, 1998; Marcuse, 1989, 1993, 1996; Marcuse \& Van Kempen, 2000; Van der Wusten \& Musterd, 1998; Van Kempen \& Murie, 2009).

Más recientemente, Thomas Maloutas (2012) ha ido más allá, apuntando que la segregación residencial es un fenómeno que depende extremadamente del contexto, respecto del cual, además de los aspectos económicos estructurales y las políticas redistributivas, es necesario tener en cuenta otros elementos estrictamente locales; por ejemplo, las características del mercado inmobiliario, la importancia de la solidaridad familiar o incluso la propia estructura territorial de la ciudad estudiada. En definitiva, según este autor, debe entenderse que la correlación entre desigualdad social y segregación residencial no es igual en todas las ciudades. En este sentido, Kuniko Fujita (2012) propone clasificar cada caso según una tipología en la que se van combinando los grados de desigualdad y de segregación que presenta cada ciudad. Así, el patrón "desigual-segregado" se correspondería con el modelo teórico de ciudad dual y es definitivamente más propio de las ciudades norteamericanas o latinoamericanas. En cambio, las ciudades europeas responden más a lógicas de "desigual-no segregado" o de "no desigual-segregado", mientras que ciudades asiáticas, como Tokio o Taipei, siguen más bien una pauta "no desigual-no segregado". 


\section{Aspectos metodológicos y fuentes de datos}

Para llevar a cabo este estudio, se ha analizado en primer lugar la transición industrial-posindustrial y la evolución tanto de la estructura ocupacional como de las desigualdades de renta en el área metropolitana de Barcelona, teniendo como referencia el periodo comprendido desde mediados de los ańos ochenta del siglo xx hasta el año 2011. En esta parte del análisis se han utilizado las fuentes de datos que ofrecen las series de información más extensas para la demarcación de Barcelona, tanto encuestas como información censal. En relación con las encuestas, por un lado, se ha utilizado la Encuesta de Población Activa (EPA) elaborada por el Instituto Nacional de Estadística (INE), que provee de información para la provincia de Barcelona; y, por otro lado, también se ha hecho uso de la Encuesta de Condiciones de Vida y Hábitos de la Población (ECVHP), realizada principalmente por el Institut d'Estudis Regionals i Metropolitans de Barcelona (IERMB) ${ }^{2}$ y que ofrece información estadísticamente significativa para el área metropolitana de Barcelona. ${ }^{3}$ El tipo de análisis realizado a partir de los datos de ambas encuestas ha sido meramente descriptivo, al mismo tiempo que se han construido indicadores estandarizados de desigualdad social, como el coeficiente de Gini o la ratio S80/S20, poniendo en valor sobre todo el carácter evolutivo de la información.

Más allá de la información de las encuestas, también se ha realizado una cartografía $^{4}$ evolutiva de la distribución territorial de las ocupaciones laborales de los residentes en el área metropolitana de Barcelona. El análisis ha sido efectuado a nivel de sección censal a partir de los datos del Censo de Población y Vivienda elaborado por el Instituto Nacional de Estadística (INE) que cubre el periodo 19912011. Cabe destacar que los datos del Censo de Población y Vivienda 2011 han sido sometidos a un tratamiento estadístico, llevado a cabo por el IERMB, para corregir la falta de información en algunas secciones censales. ${ }^{5} \mathrm{La}$ información censal se ha utilizado también para calcular el indice de segregación (Is) de algunos grupos ocupacionales. Este indicador puntúa de 0 (ausencia de segregación) a 1 (máxima segregación) e indica la proporción del grupo social estudiado que debería cambiar de residencia para obtener una distribución residencial igualitaria en todo el ámbito de estudio (Duncan \& Duncan, 1955a; Massey \& Denton, 1988). Además, se ha realizado un análisis territorial de las pautas de localización residencial de los

2 En las últimas dos ediciones de esta operación estadística también ha colaborado el Institut d'Estadística de Catalunya (IDESCAT), pasando a ser estadística oficial. Para más información sobre esta encuesta, véase: http://www.enquestadecondicionsdevida.cat.

3 Integrada por los 36 municipios que forman parte de la administración pública denominada Àrea Metropolitana de Barcelona, constituida en 2011 a partir de la Llei 31/2010 aprobada en el Parlament de Catalunya.

4 Los mapas incluidos en este artículo se han realizado mediante el software ARCGIS.

5 Este procedimiento de imputación se ha realizado siguiendo un método que preserva la variabilidad de las variables y la correlación entre ellas a partir de una secuencia de modelos de regresión logística (Raghunathan, Lepkowski, Van Hoewyk \& Solenberger, 2001). La aplicación se ha realizado a partir de la macro de SAS Iveware desarrollada por la Universidad de Michigan (http://www.isr.umich.edu/src/smp/ive/). 
grupos ocupacionales objeto de estudio, basado en los índices global y local de Moran. Estos indicadores permiten determinar si existe una pauta de agrupación o de dispersión en la localización residencial de estos grupos a lo largo de la metrópoli, o si, por el contrario, su comportamiento residencial no sigue ningún patrón territorial (Anselin, 1995; Anselin, Syabri \& Kho, 2006).

Con la información analizada, validar el modelo de ciudad dual implicaría, por un lado, un aumento en la estructura ocupacional de la proporción de trabajadores de servicios cualificados y de trabajadores de servicios no cualificados, así como un aumento del coeficiente de Gini. Y, por otro lado, a nivel socioespacial, se debería registrar un aumento del índice de segregación y una tendencia creciente de localización residencial agrupada que comportara, a su vez, un aumento progresivo de la ocupación del espacio urbano por parte de estos dos grupos ocupacionales.

\section{La transformación de la estructura ocupacional de la Barcelona metropolitana en el tránsito posindustrial}

Durante los primeros años de la década de los ochenta, la metrópoli de Barcelona inició su etapa posindustrial. En esos años, por primera vez, las ocupaciones en el sector servicios superaron en peso relativo a las del sector industrial (figura 1). Como es sabido, este proceso de terciarización del sistema productivo no es particular de Barcelona, sino que al mismo tiempo ya se estaba produciendo en el resto de grandes ciudades espańolas (Méndez \& Caravaca, 1993), eso sí, con cierto retraso con respecto a otros países industrializados en los que esta transición productiva ya había comenzado en los años setenta (Bell, 1976).

Como refleja la figura 1, en el caso de Barcelona se pueden identificar dos etapas diferenciadas de terciarización en las últimas décadas. La primera se inicia prácticamente con la transición democrática y acaba con la crisis económica posolímpica que tiene lugar aproximadamente entre 1992 y 1995. Este periodo se caracteriza sobre todo por un rápido crecimiento económico de la ciudad, que se ve reforzado por el desarrollo del sector público, la nominación de Barcelona como sede de los Juegos Olímpicos de 1992 y el ingreso de Espańa en las Comunidades Europeas (Trullén, 1998). Después de prácticamente una década de relativa estabilidad, la segunda etapa de terciarización se inició con el cambio de siglo. Esta etapa también vino marcada, en un primer momento, por un periodo de crecimiento económico que duró hasta 2007, en esta ocasión espoleado por el relevante incremento de la actividad densa en conocimiento que se produjo en la capital catalana durante esos ańos, y por la consolidación de la internacionalización de la economía barcelonesa (Trullén, Lladós \& Boix, 2002). Además, todo ello coincide con el último gran boom inmobiliario espańol (Rodríguez, 2006), el cual también ayudó a dinamizar la economía del área metropolitana de Barcelona. A partir de 2008, en cambio, estalla la burbuja inmobiliaria y se inicia la crisis económica y financiera. Sin embargo, a pesar de ello y a diferencia de lo que había ocurrido en la década de los noventa, esta vez el proceso de terciarización no se detiene ante el cambio de ciclo económico, sino que continúa e incluso gana intensidad. En este sentido, es preciso apuntar que en la crisis actual gran parte de la destrucción de ocupación que se produce en 
el área metropolitana de Barcelona se localiza en el sector industrial, además de la construcción (Sarasa et al., 2013).

Otro aspecto que resulta relevante destacar en relación a los dos periodos de terciarización que ha vivido la metrópolis de Barcelona es que, si bien en el primero la transformación de la base productiva es adoptada por la ciudad prácticamente por inercia, en el segundo se busca ya esta transformación de manera proactiva desde las políticas urbanas, siendo el proyecto del 22@en el barrio del Poblenou el paradigma de esta reorientación económica de la ciudad ${ }^{6}$ (Trullén, 2001; Trullén et al., 2002).

\section{Figura I | Evolución de los sectores de actividad. Provincia de Barcelona, 1976-2014. Población ocupada (\%)}

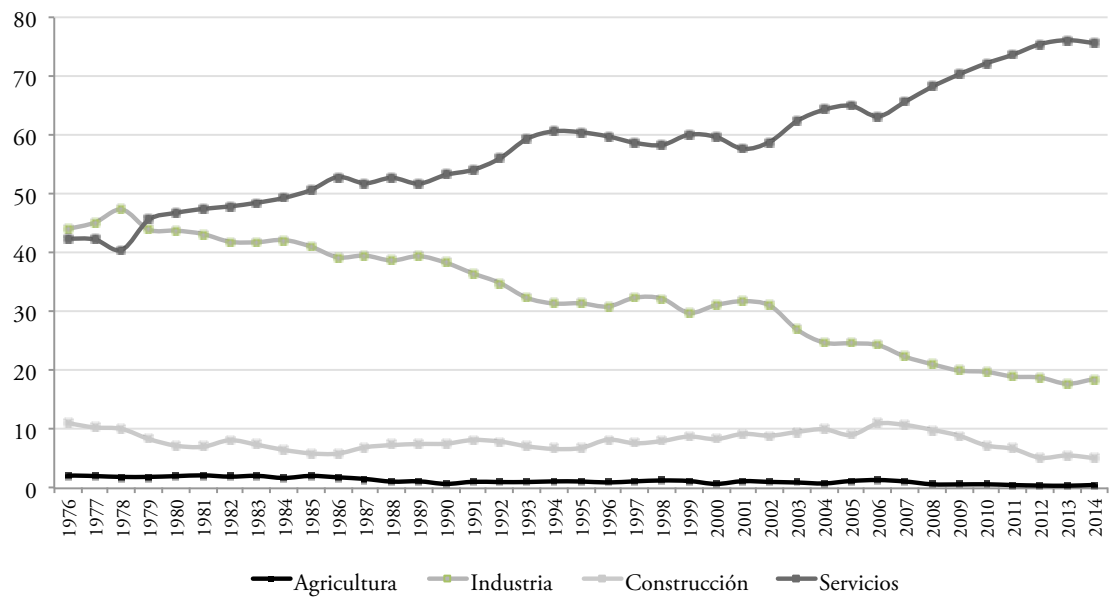

FUENTE IERMB, A PARTIR DE DATOS DE LA ENCUESTA DE POBLACIÓN ACTIVA, BASE POBLACIONAL 200 I Y $20 \mathrm{I}$ I, INE

Como se ha explicado anteriormente, son diversos los autores que relacionan este tránsito industrial-posindustrial con la polarización de la estructura social y el aumento de las desigualdades sociales, particularmente en las grandes ciudades. De hecho, Oriol Nel.lo (1994), a mediados de la década de los noventa, justo después del primer proceso intenso de terciarización que se produjo en la economía barcelonesa, ya apuntaba que la población metropolitana de Barcelona comenzaba a sufrir algunos efectos socioeconómicos, particularmente en el caso de los trabajadores que se veían afectados por la reestructuración industrial. Entre los efectos que señala este autor, destacan la pérdida de ingresos generalizada, la dificultad para encontrar trabajo, la pérdida de derechos laborales o la pérdida de cohesión e identidad de las comunidades y grupos sociales vinculados a la actividad desaparecida.

El Plan 22@ es un proyecto de regeneración urbana diseńado para transformar la principal zona industrial de la ciudad, situada en el barrio del Poblenou, en un distrito de innovación económica en el cual priorizar la localización de empresas vinculadas a los sectores biomédico, los new media, el diseńo, etc. (Ajuntament de Barcelona, 2011). 
Pero, ¿qué ha ocurrido desde una óptica más general? ¿Cómo han evolucionado la estructura social y las desigualdades sociales en el área metropolitana de Barcelona durante todo el periodo de desindustrialización?

El primer aspecto relevante que emerge de los datos analizados es que efectivamente la estructura ocupacional de la metrópoli barcelonesa se ha terciarizado y polarizado en las dos últimas décadas. La figura 2 muestra cómo la categoría ocupacional que más ha crecido entre 1985 y 2011 es la de trabajadores semicualificados de servicios, cuya proporción sobre el total de población ocupada pasó del 13,7\% en 1985 al 27,0\% en 2011. Además, los profesionales y directivos (trabajadores de servicios cualificados) siguieron una moderada tendencia al alza durante el mismo periodo, con un fuerte incremento en los últimos ańos, que entre 1985 y 2011 significó pasar del 26,1\% al 35,9\%. Por el contrario, la categoría que más se redujo durante el mismo periodo fue la de los trabajadores industriales semicualificados y los de rutina. Este tipo de trabajadores pasó de ser la categoría ocupacional mayoritaria en $1985(27,7 \%)$ a ser prácticamente la menos relevante en 2011 (12,0\%), junto con los pequeños empresarios y autónomos (10,8\%). Sin duda, la transición a una estructura productiva posindustrial y la consecuente alteración en la demanda de mano de obra constituye la principal causa de esta tendencia hacia la terciarización y la polarización de la estructura ocupacional barcelonesa.

FIGURA 2 | Evolución de la estructura ocupacional. Área metropolitana de Barcelona, 1985-2011. Población ocupada. 1985=100

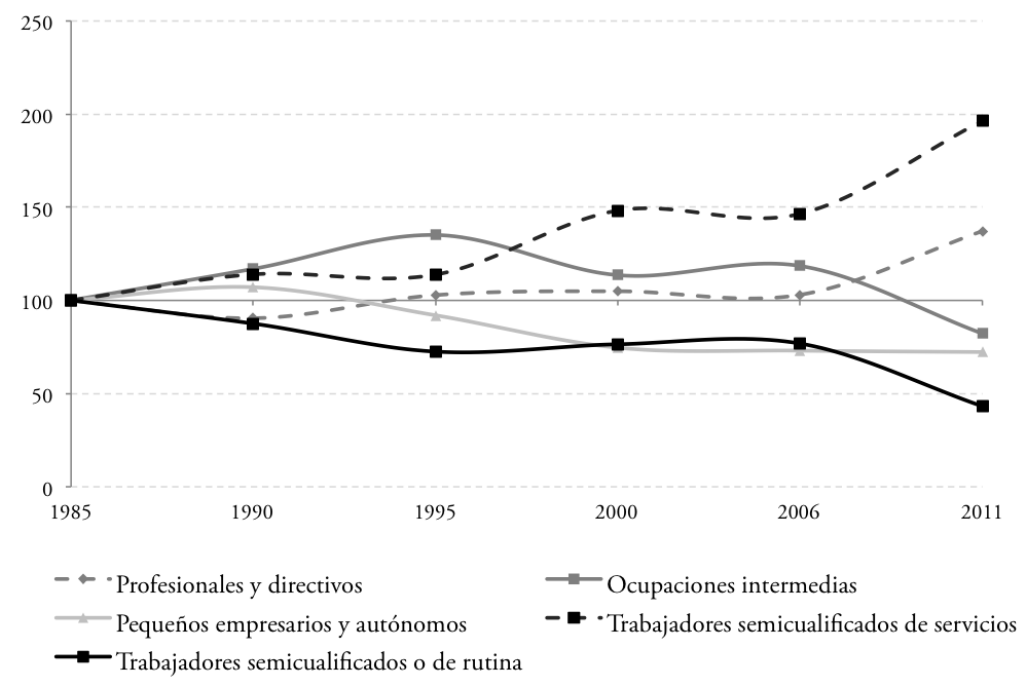

FUENTE IERMB, ENCUESTA DE CONDICIONES DE VIDA Y HÁBITOS DE LA POBLACIÓN, I985-2000; IDESCAT / IERMB, ENCUESTA DE CONDICIONES DE VIDA Y HÁBITOS DE LA POBLACIÓN, 2OO6-2OI I

Los resultados de la figura 2 arrojan además otros matices interesantes si se relacionan con cada contexto económico.

En primer lugar, coincidiendo con el primer periodo de terciarización, entre 1985 y 1995 se produjo un incremento relevante de las ocupaciones intermedias 
(del 17,5\% al 23,7\%), una categoría constituida principalmente por administrativos, técnicos de apoyo y capataces de la industria. La expansión del sector público generada durante estos años por la reinstauración de la Generalitat de Catalunya -la cual supone precisamente la capitalidad de Barcelona, que hasta entonces tenía una proporción de funcionarios irrisoria- y el desarrollo de las administraciones locales democráticas, constituyeron un factor clave en este sentido. Por otro lado, durante el mismo periodo también se dio un descenso significativo de los trabajadores semicualificados industriales, poniendo de manifiesto la intensidad de esta primera etapa de terciarización económica en el área metropolitana de Barcelona.

En segundo lugar, el periodo 1995 y 2006, que coincide en parte con el inicio de la segunda fase de terciarización, fue el más estable para la estructura ocupacional de Barcelona, tan solo alterada por el fuerte incremento de los trabajadores semicualificados de servicios que se dio con el cambio de siglo.

Finalmente, la dinámica de destrucción masiva de ocupación que tuvo lugar durante los primeros ańos de la crisis económica -a partir de 2008- acabó por reconfigurar la estructura ocupacional de Barcelona, reforzando la presencia de los trabajadores de servicios, tanto cualificados (profesionales y directores) como semicualificados, al mismo tiempo que se redujeron claramente las proporciones de ocupaciones intermedias y de los trabajadores semicualificados o de rutina, relacionados mayoritariamente con la industria. De hecho, no fue solo la dinámica de destrucción de ocupación la que generó estas tendencias, sino que, además, ellas se vieron reforzadas por el hecho de que la poca ocupación que se creó en este contexto de recesión económica se dio en el sector servicios, siendo los trabajadores semicualificados de servicios los únicos que aumentaron en número (Sarasa et al., 2013). El resultado de todo este proceso fue la configuración de una estructura ocupacional dualizada, dominada, por un lado, por profesionales y directivos $(35,9 \%)$; y por otro lado, por trabajadores semicualificados de servicios $(27,0 \%)$, dos grupos ocupacionales con condiciones laborales muy desiguales.

\section{La evolución de las desigualdades sociales en Barcelona: del proceso cohesionador a la fractura de la crisis}

Tal como apuntan los planteamientos teóricos del modelo de ciudad dual expuestos anteriormente, la evolución que ha seguido la estructura ocupacional del área metropolitana de Barcelona, especialmente durante el segundo periodo de terciarización, marcado por el predominio adquirido por los trabajadores cualificados y no cualificados de servicios, podría constituir una buena base explicativa para una tendencia creciente de las desigualdades sociales. En la medida en que los obreros industriales y los empleados de oficina cualificados constituyen el grueso de los estratos intermedios de renta, ${ }^{7}$ su pérdida de peso en la estructura ocupacional ha

7 En Cataluńa, los obreros semicualificados y los empleados en ocupaciones rutinarias constituyen más del $40 \%$ de los efectivos del estrato intermedio de la distribución de la renta, seguidos en importancia por el grupo de encargados y administrativos cualificados (más del 20\%), los autónomos y los pequeños empresarios (10\%), y también de los desocupados de larga duración (10\%). De hecho, entre el $70 \%$ u $80 \%$ de la clase trabajadora se sitúa en este estrato intermedio de renta (Sarasa et al., 2013). 
comportado en muchos países el encogimiento de estos estratos, dando pie a la alarma que advierte de una desaparición de las clases medias (en términos de renta), con los peligros que ello puede comportar tanto para el consumo y el crecimiento económico, como para la cohesión social y la gobernanza de las ciudades. Esta tendencia, sin embargo, no se ha producido en el área metropolitana de Barcelona de forma clara hasta el estallido de la actual crisis económica.

En la figura 3 se puede observar cómo entre los años 1985 y 1995, coincidiendo con el primer periodo de terciarización y el notable incremento de las ocupaciones intermedias, se produjo un importante crecimiento relativo del estrato intermedio de renta (del 54,9\% pasó al 70,8\%). Este incremento tuvo lugar tanto a expensas del estrato de rentas altas, que cayó del $28,9 \%$ al $22,1 \%$, como del estrato de rentas bajas, con un descenso del 16,3\% al 7,1\%. Es preciso tener en cuenta que durante la década de los ochenta, los primeros gobiernos democráticos reforzaron las prestaciones de paro, las pensiones de vejez y los seguros por enfermedad (Moreno \& Sarasa, 1992), lo que contribuyó a una reducción relevante de las rentas bajas.

Con el inicio del segundo periodo de terciarización y durante la etapa de crecimiento económico anterior a la actual crisis económica, el proceso cohesionador de ensanchamiento del estrato intermedio de renta tocó techo, al mismo tiempo que el estrato de rentas bajas tendía a aumentar de forma moderada (del 7,1\% en 1995 al $10,8 \%$ en 2006). No obstante, como se ha dicho anteriormente, no fue hasta 2011, en pleno contexto de crisis, que se produjo una disminución drástica del estrato intermedio de renta, que pasó del 69,0\% en 2006 al 63,0\% en 2011, ante el aumento de los estratos de rentas altas (del 20,3\% en 2006 al 24,1\% en 2011) y de rentas bajas (del 10,8\% en 2006 al 12,9\% en 2011).

FIGURA 3 | Estratificación de renta familiar disponible. Área metropolitana de Barcelona, 1985-2011. Población de 18 años y más (\%)

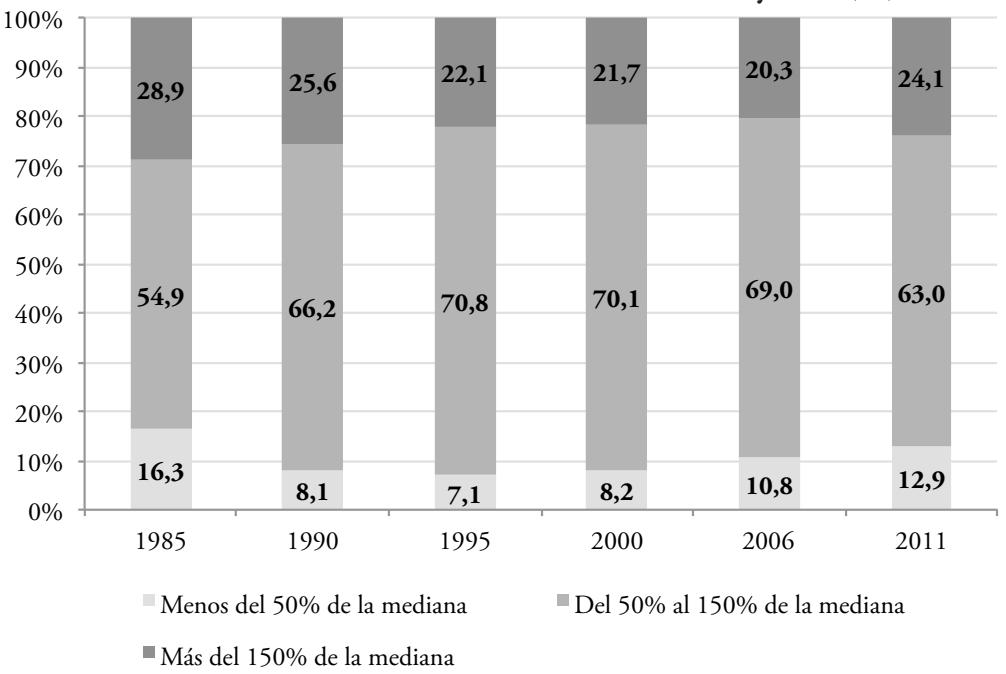

FUENTE IERMB, ENCUESTA DE CONDICIONES DE VIDA Y HÁBITOS DE LA POBLACIÓN, I985-200O; IDESCAT / IERMB, ENCUESTA DE CONDICIONES DE VIDA Y HÁBITOS DE LA POBLACIÓN, 2006-2OI I 
Los principales indicadores de desigualdad de renta corroboran también estas tendencias, al mismo tiempo que permiten añadir algunos matices. En primer lugar, el coeficiente de Gini marca una disminución incesante de la desigualdad social en el área metropolitana de Barcelona entre 1985 y 2006, pasando del 0,397 al 0,293, y un importante crecimiento de la misma en el contexto de la crisis (2011), situándose en un 0,327, un valor similar al registrado en el año 1995 (0,325). En segundo lugar, las ratios entre los deciles extremos y la mediana (S90/S50 y S50/S10) indican que este último aumento de la desigualdad social se explica principalmente por la caída de ingresos de la población con rentas más bajas, en gran parte causada por la masiva pérdida del empleo ocasionada por la crisis.

No obstante, la figura 4 revela también que este aumento del peso de las rentas bajas no es únicamente un efecto de la crisis, sino que más bien se trata de una tendencia que se inicia con el cambio de siglo y que se intensifica con la recesión económica. Por lo tanto, ella se origina en el segundo periodo de terciarización, en un contexto de fuerte crecimiento económico y en el que aumentan considerablemente las ocupaciones semicualificadas de servicios. Además, durante este periodo, el área metropolitana de Barcelona recibe un volumen espectacular de inmigrantes extranjeros, siendo, junto a Madrid, la principal puerta de entrada migratoria estatal (Fullaondo, 2007). La fuerte llegada de población inmigrada es otro de los factores que ayuda a entender el aumento de las rentas bajas en este periodo, en la medida en que este colectivo es de los que más sufre la precariedad laboral y los bajos salarios, insertándose mayoritariamente en ocupaciones de bajo rango y con escasa movilidad ascendente (Bernardi, Garrido \& Miyar, 2011; Martín, López-Roldán \& Molina, 2011). A pesar de ello, se puede decir que, a grandes rasgos, la metrópoli de Barcelona tendió a reducir la desigualdad social hasta el inicio de la actual crisis económica, momento en que se ha vuelto a incrementar significativamente.

FIGURA 4 | Evolución de indicadores de desigualdad de renta (1985=100). Área metropolitana de Barcelona, 1985-2011

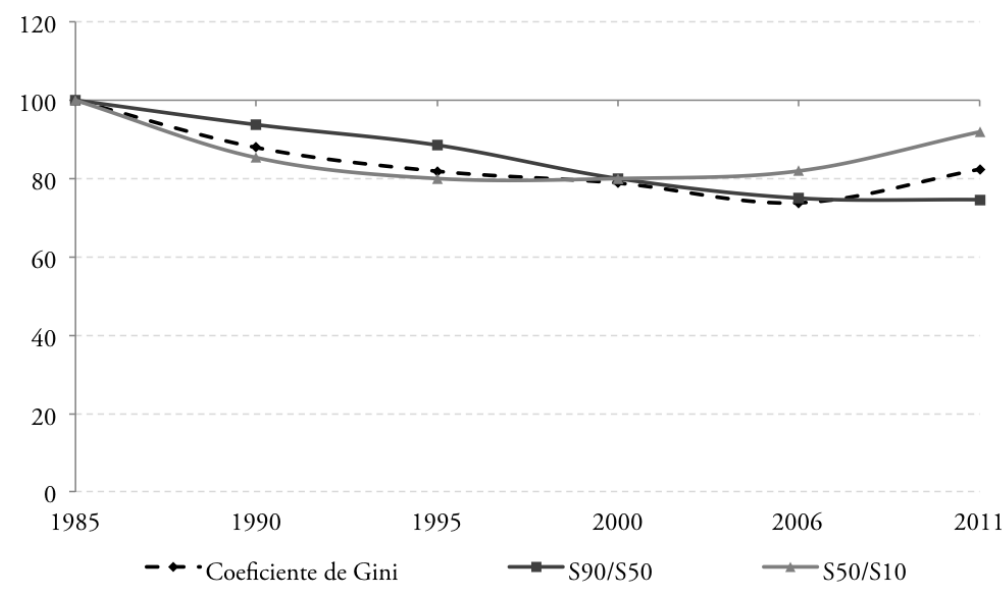

FUENTE IERMB, ENCUESTA DE CONDICIONES DE VIDA Y HÁBITOS DE LA POBLACIÓN, I985-2000; IDESCAT / IERMB, ENCUESTA DE CONDICIONES DE VIDA Y HÁBITOS DE LA POBLACIÓN, 2006-2OI I 
En este sentido, la metrópoli de Barcelona constituiría un caso atípico, si adoptamos como referencia los parámetros teóricos del modelo de ciudad dual, ya que a pesar de la terciarización del sistema productivo y la reestructuración ocupacional, la desigualdad social se redujo entre 1985 y 2006. De hecho, el aumento de la desigualdad social de los últimos años no se puede explicar tampoco por estos factores, a pesar de que, como se ha visto anteriormente, durante este periodo sí que se puede identificar un pequeño efecto de cambio de tendencia que se podría asociar a la desindustrialización. No obstante, todo indica que el repunte de las desigualdades sociales tiene más que ver con el dramático incremento del paro que se produce con la llegada de la crisis económica a partir de 2008.

Son diversos los elementos que pueden explicar la particularidad del caso de Barcelona. En primer lugar, hay que considerar el desarrollo del Estado de Bienestar español con la instauración de la democracia y sus efectos redistributivos en términos de renta, pese a sus limitaciones con respecto a otros países del norte europeo (Moreno \& Sarasa, 1992). En segundo lugar, resulta también inapelable el espectacular crecimiento de población ocupada que se produce en la conurbación de Barcelona durante todo este periodo, pasando aproximadamente de 1.300 .000 ocupados en 1985 a casi 2.600 .000 ocupados en 2006 (Trullén et al., 2002). Además, como se ha visto anteriormente, durante el primer periodo de terciarización este crecimiento de ocupación favoreció sobre todo el crecimiento de ocupaciones intermedias, lo cual explicaría en gran parte la mejora de la cohesión social durante estos primeros ańos, hasta mediados de los noventa. Sin embargo, a partir de 1995 y hasta el inicio de la crisis económica, un periodo en el que empezaba a gestarse la polarización de la estructura ocupacional, el fenómeno que más ayuda a explicar la disminución de las desigualdades sociales es el aumento de la actividad laboral femenina (figura 5). Este fenómeno contribuye a matizar los efectos del aumento de la ocupación semicualificada de servicios en la reconfiguración de la estructura ocupacional que se produjo durante este periodo, en la medida en que aumentaron los hogares de dobles ingresos. Más del 70\% de la ocupación semicualificada de servicios era femenina, y la mayoría de estas mujeres residía en hogares de dobles ingresos, reduciendo su riesgo de pobreza y la desigualdad en la distribución de la renta. Por lo tanto, en el área metropolitana de Barcelona, la polarización ocupacional no se tradujo en una mayor desigualdad de renta mientras la economía crecía, porque el sesgo de género presente en las nuevas ocupaciones contribuyó a aumentar los ingresos de los hogares donde antes las mujeres eran inactivas, haciendo que la dispersión de la renta se redujera. 
FIgURA 5 | Evolución de la actividad laboral según sexo. Área metropolitana de Barcelona, 1985-2011. Población en parejas casadas o cohabitantes $(\%)$

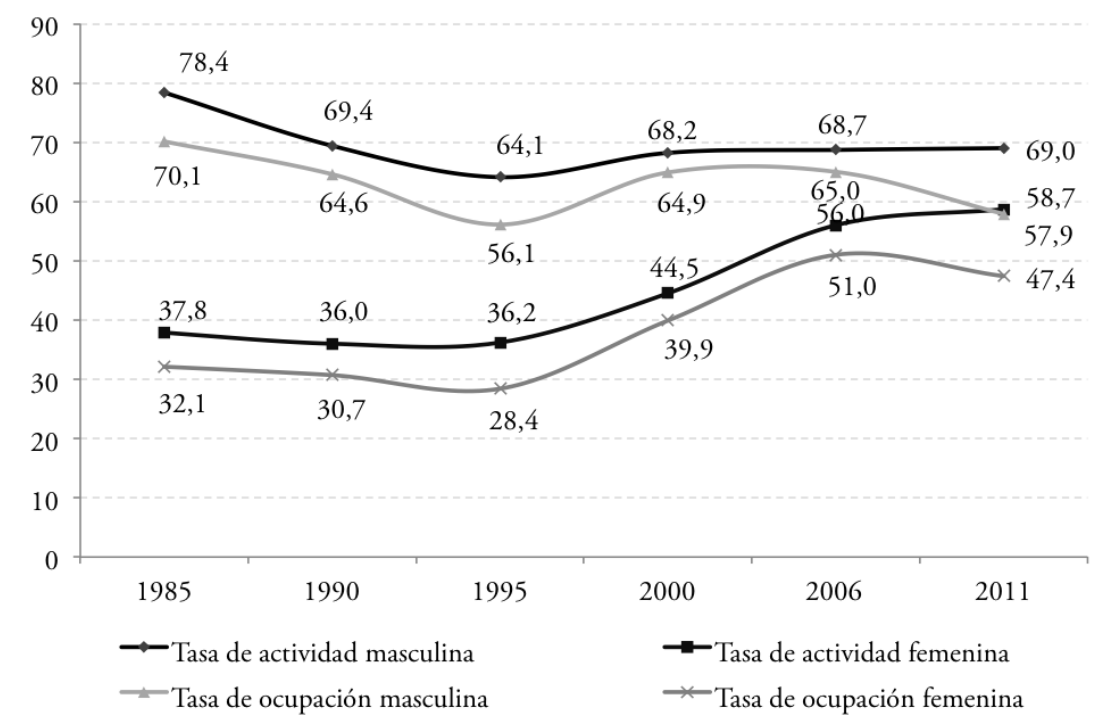

FUENTE IERMB, ENCUESTA DE CONDICIONES DE VIDA Y HÁBITOS DE LA POBLACIÓN, I985-2000; IDESCAT / IERMB, ENCUESTA DE CONDICIONES DE VIDA Y HÁBITOS DE LA POBLACIÓN, 2OO6-2OI I

La evolución de la dinámica socioterritorial en el área metropolitana de Barcelona desde una perspectiva socioeconómica

La polarización ocupacional que se ha consolidado durante las últimas décadas en la estratificación socioeconómica de la metrópoli de Barcelona se ha trasladado también a su estructura residencial, incluso de manera más evidente (tabla 1). Si se atiende a la categoría ocupacional mayoritaria entre la población ocupada residente en cada una de las secciones censales que componen esta aglomeración urbana, se puede observar que tanto los profesionales y directivos como los trabajadores semicualificados de servicios son los dos perfiles ocupacionales que han ido ganando cada vez más presencia en la estructura residencial de Barcelona, en detrimento sobre todo de los trabajadores semicualificados de la industria.

Para conocer mejor el comportamiento espacial de estos dos perfiles sociales, se han analizado dos de los principales indicadores de segregación residencial (tabla 2). Los resultados del índice de segregación (Is) desvelan, por un lado, que la localización residencial de los profesionales y directivos sigue una pauta más segregada que la de los trabajadores semicualificados de servicios. Este resultado no sorprende en absoluto, ya que -como se ha puesto de manifiesto en diferentes estudios- tanto en las ciudades norteamericanas como en las europeas las clases con más recursos se segregan más que las clases populares (Duncan \& Duncan, 1955b; Maloutas, 2012; Préteceille, 1995; Uyeki, 1964; White, 1984). No obstante, se puede observar también que la 
segregación residencial de los profesionales y directivos perdió intensidad durante la década de los noventa (de 0,415 en 1991 a 0,342 en 2001), mientras que en la última década se ha mantenido prácticamente al mismo nivel. Por el contrario, los trabajadores semicualificados de servicios han ido aumentando ligeramente su nivel de segregación residencial conforme han ido dominando una proporción mayor del territorio metropolitano barcelonés (de 0,097 en 1991 a 0,208 en 2011).

TABLA I | Categoría ocupacional predominante entre la población ocupada residente en cada sección censal.

Área metropolitana de Barcelona, 1991-2011

\begin{tabular}{|c|c|c|c|c|c|c|}
\hline & \multicolumn{3}{|c|}{ SECCIONES CENSALES (\%) } & \multicolumn{3}{|c|}{ SUPERFICIE $\left(\mathrm{KM}^{2}\right)$} \\
\hline & I99I & $200 I$ & $201 I^{*}$ & I99I & $200 I$ & 2OII* \\
\hline Áreas de profesionales y directivos & 9,8 & 29,4 & 35,4 & 19,7 & 51,5 & 61,6 \\
\hline Áreas de ocupaciones intermedias & 23,1 & 17,5 & 16,8 & 15,9 & 26,3 & 26,5 \\
\hline $\begin{array}{l}\text { Áreas de trabajadores semicualificados de } \\
\text { servicios }\end{array}$ & 13,5 & 17,5 & 42,0 & 18,2 & 15,1 & 46,6 \\
\hline $\begin{array}{l}\text { Áreas de trabajadores semicualificados de } \\
\text { la industria }\end{array}$ & 51,5 & 30,9 & 2,9 & 71,3 & 45,3 & 6,8 \\
\hline Áreas de trabajadores no cualificados & 1,5 & 2,9 & 2,9 & 2,2 & 2,0 & 2,8 \\
\hline Áreas mixtas & 0,7 & 1,8 & 0,0 & 0,4 & 1,3 & 0,0 \\
\hline Total & 100,0 & 100,0 & 100,0 & 127,6 & 141,5 & 144,3 \\
\hline
\end{tabular}

FUENTE INE, CENSO DE POBLACIÓN Y VIVIENDA, I 99I-2OI I

* DATOS FAlTANTES IMPUTAdos POR EL IERMB A PARTIR DEL SOFTWARE IVEWARE.

Por otro lado, el índice global de Moran (I) indica también que los profesionales y directivos presentan un patrón de localización residencial mucho más agrupado que los trabajadores semicualificados de servicios. De hecho, hay que decir que durante la década de los noventa, la localización residencial de los profesionales y directivos es extremadamente agrupada, con puntuaciones, tanto en 1991 como en 2001, por encima del 0,8 . Sin embargo, este patrón pierde ligeramente consistencia a partir del cambio de siglo $(0,637)$. Lo mismo ocurre con los trabajadores semicualificados de servicios, que aparecen en el año 2011 menos agrupados residencialmente que en años anteriores, rompiendo radicalmente con la tendencia creciente que registran durante la década de los noventa.

TABLA 2 Índice de Segregación y índice global de Moran (I) según categoría ocupacional. Área metropolitana de Barcelona, 1991-2011

\begin{tabular}{|l|c|c|c|c|c|c|}
\hline & \multicolumn{4}{|c|}{} & \multicolumn{3}{c|}{$\begin{array}{c}\text { TRABAJADORES } \\
\text { SEMICUALIFICADOS DE } \\
\text { SERVICIOS }\end{array}$} \\
\cline { 2 - 7 } & I99I & $\mathbf{2 0 0 I}$ & $\mathbf{2 0 I} \mathbf{I}^{*}$ & I99I & $\mathbf{2 0 0 I}$ & $\mathbf{2 O I}^{*}$ \\
\hline Índice de Segregación (IS) & 0,415 & 0,342 & 0,349 & 0,097 & 0,123 & 0,208 \\
\hline Índice Global de Moran (I) & 0,582 & 0,602 & 0,545 & 0,316 & 0,413 & 0,129 \\
\hline
\end{tabular}

FUENTE INE, CENSO DE POBLACIÓN Y VIVIENDA, I 99I-2OI I.

* DATOS FAltantes imputados POR El iERMB a PARTir DEL SOFTWARE IVEWARE. 
Las figuras 6, 7 y 8 presentan en negro cómo han evolucionado las zonas residenciales en las que los profesionales y directivos registran una autocorrelación espacial alta (alta-alta), es decir, donde se agrupan residencialmente. En ellas se puede observar cómo, a principios de la década de los noventa, la pauta residencial de este grupo ocupacional se sitúa en sintonía con el eje que establece la avenida Diagonal en Barcelona, tanto dentro del municipio - donde destaca la denominada zona alta de la ciudad y el Eixample-, como en su proyección más allá de la ciudad central, llegando a los municipios de Esplugues de Llobregat y Sant Just Desvern. Además, este grupo ocupacional también aparece ubicado de forma agrupada en una amplia zona del municipio de Sant Cugat del Vallès, como si se tratara de una prolongación de la zona alta de Barcelona al otro lado de la sierra de Collserola. Los mapas muestran que, con el paso de los años, la presencia de este grupo ocupacional se expande por Sant Cugat del Vallès y por la ciudad central, donde va ocupando zonas regeneradas, como la Vila Olímpica o el Front Marítim, y también zonas céntricas, llegando incluso en los último años a tener una presencia relevante en determinadas zonas de Ciutat Vella. Las figuras 9, 10 y 11, en cambio, representan la evolución de la pauta residencial de los trabajadores semicualificados de servicios, los cuales -tal y como refleja el índice global de Moran (tabla 2) - no presentan un patrón de localización residencial tan agrupado como los profesionales y directivos, particularmente en los últimos años. Además, también resulta relevante la tendencia de este grupo ocupacional a abandonar las zonas céntricas del municipio de Barcelona, para reubicarse en la periferia de la propia ciudad central, o bien justo en los municipios limítrofes de Barcelona, como Santa Coloma de Gramenet o l'Hospitalet de Llobregrat, ocupando zonas residenciales de clara tradición obrera industrial.

FIgURA 6 | Índice Local de Moran (LISA) sobre la categoría ocupacional de profesionales y directivos. Área metropolitana de Barcelona, 1991

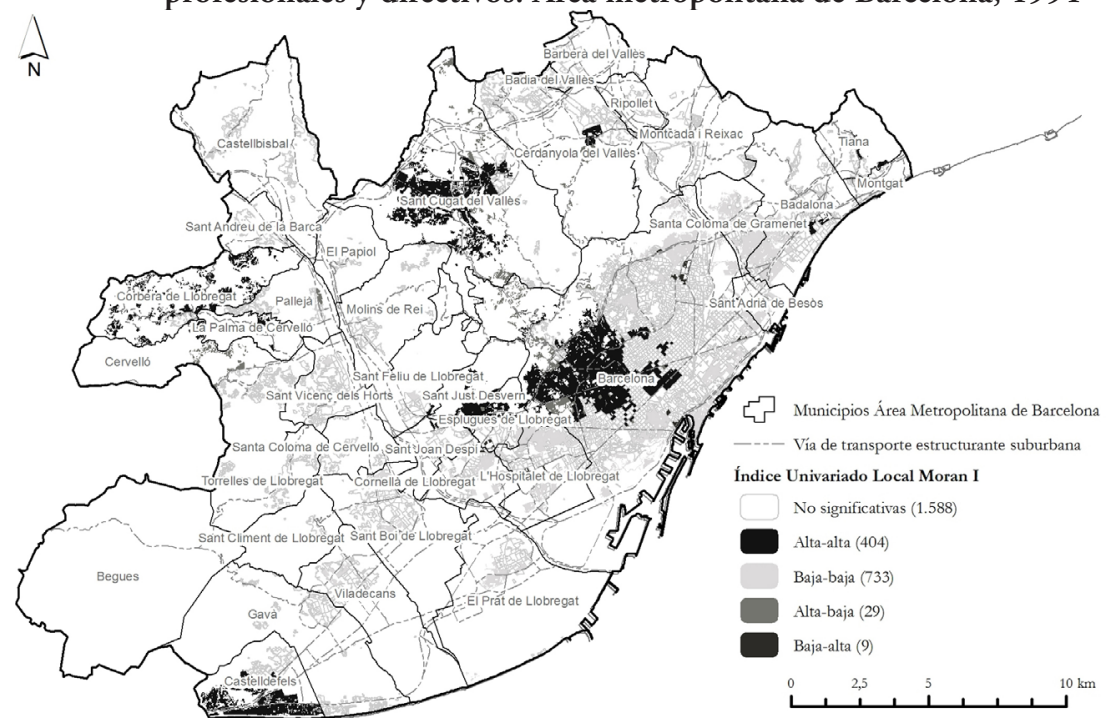

FUENTE ELABORACIÓN PROPIA. BASES CARTOGRÁFICAS: INSTITUT CARTOGRÀFIC DE CATALUNYA. DATOS CENSALES: I 99I, INSTITUTO NACIONAL DE ESTADÍSTICAS, GOBIERNO DE ESPAÑA 
FIgURA 7 | Índice Local de Moran (LISA) sobre la categoría ocupacional de profesionales y directivos. Área metropolitana de Barcelona, 2001

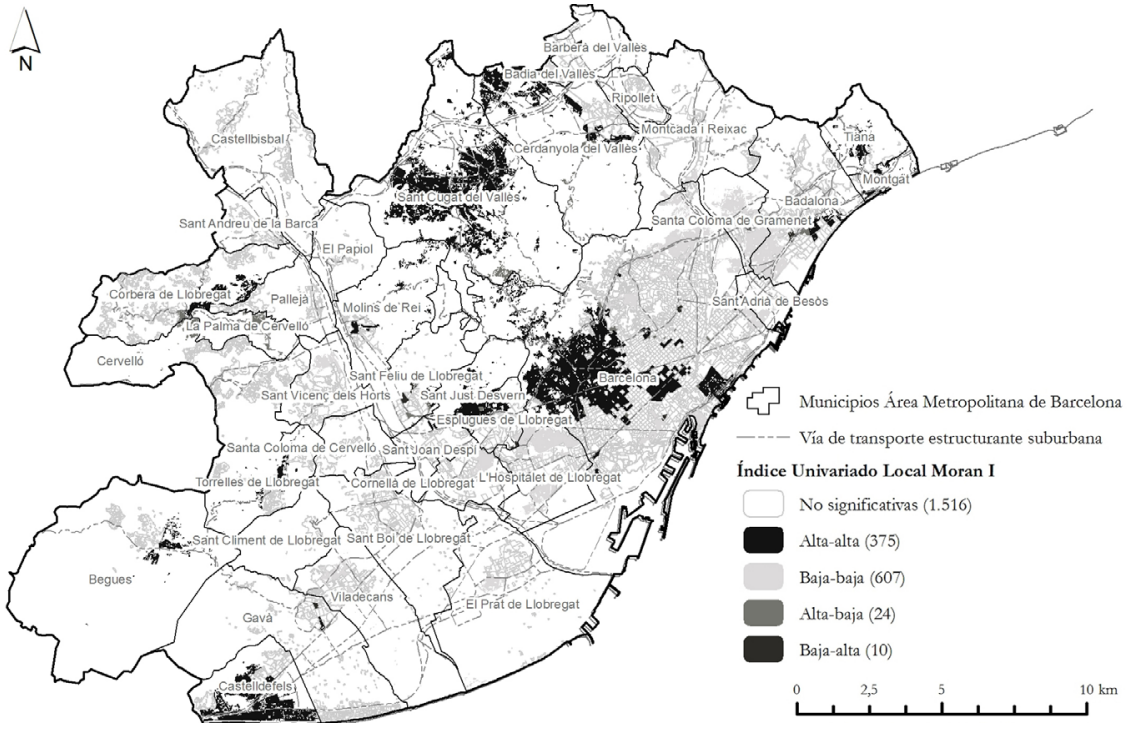

FUENTE ELABORACIÓN PROPIA. BASES CARTOGRÁFICAS: INSTITUT CARTOGRÀFIC DE CATALUNYA. DATOS CENSALES: 2OOI, INSTITUTO NACIONAL DE ESTADÍSTICAS, GOBIERNO DE ESPAŃA

FIgURA 8 | Índice Local de Moran (LISA) sobre la categoría ocupacional de profesionales y directivos. Área metropolitana de Barcelona, 2011

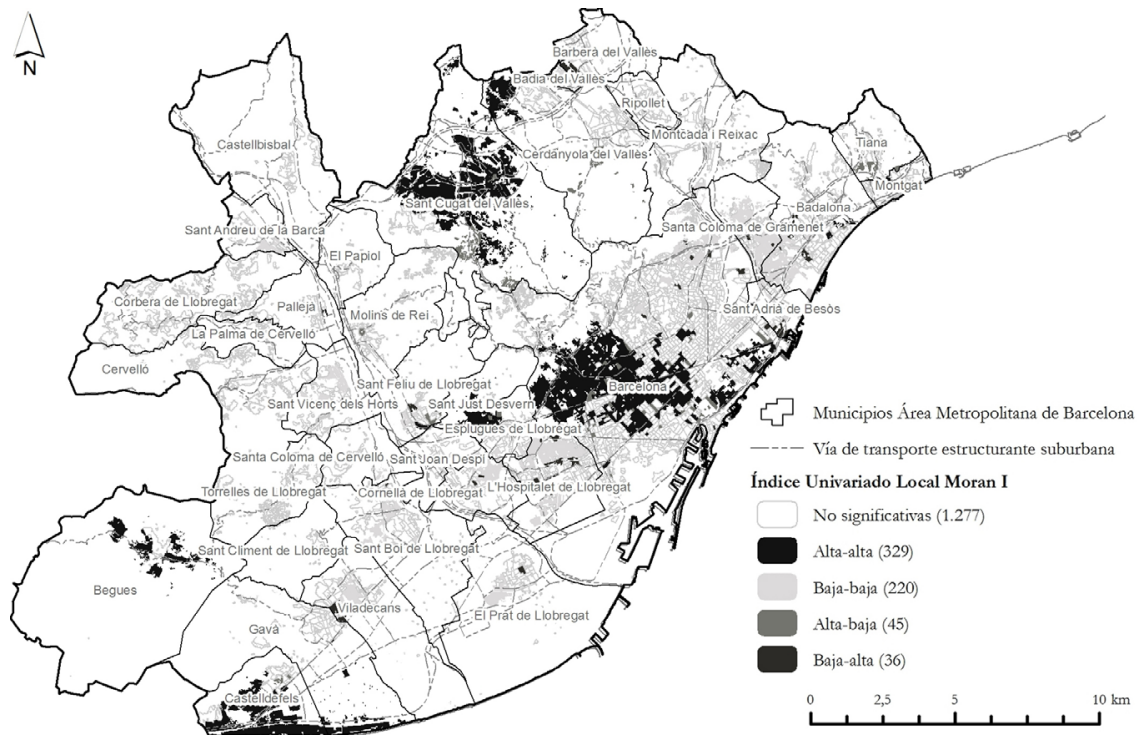

FUENTE ELABORACIÓN PROPIA. BASES CARTOGRÁFICAS: INSTITUT CARTOGRÀFIC DE CATALUNYA. DATOS CENSALES: 20 I I, INSTITUTO NACIONAL DE ESTADÍSTICAS, GOBIERNO DE ESPAÑA 
FIgURA 9 | Índice Local de Moran (LISA) sobre la categoría ocupacional de trabajadores semicualificados de servicios.

Área metropolitana de Barcelona, 1991

$\widehat{N}_{\mathrm{N}}$

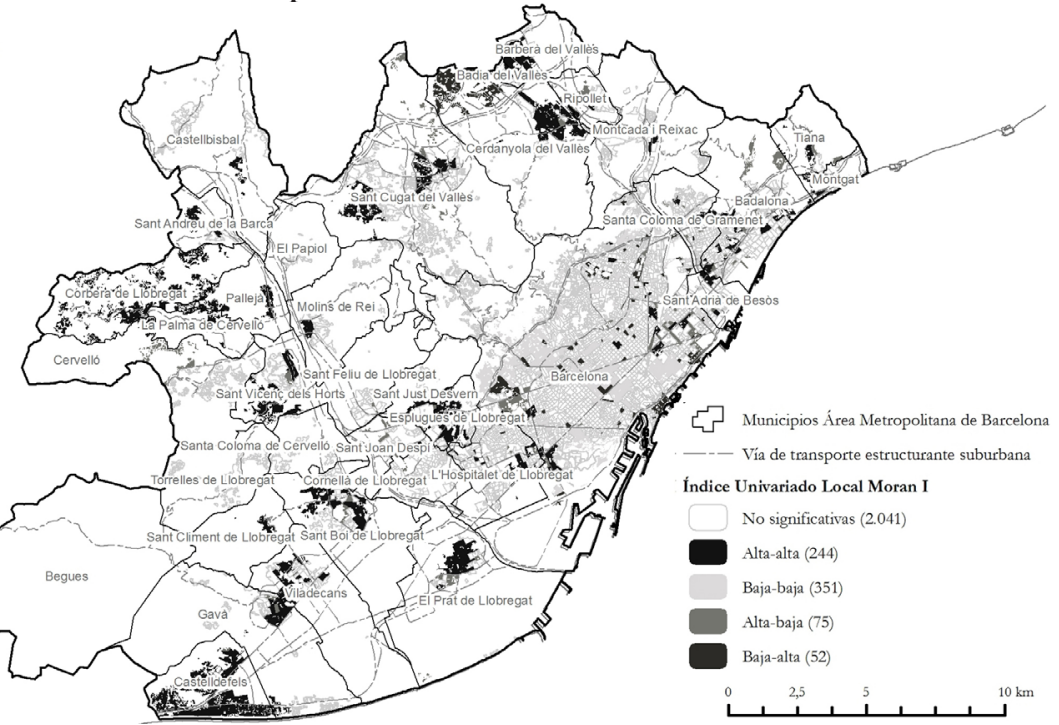

FUENTE ELABORACIÓN PROPIA. BASES CARTOGRÁFICAS: INSTITUT CARTOGRÀFIC DE CATALUNYA. DATOS CENSALES: I 99I, INSTITUTO NACIONAL DE ESTADÍSTICAS, GOBIERNO DE ESPAÑA

FIGURA io | Índice Local de Moran (LISA) sobre la categoría ocupacional de trabajadores semicualificados de servicios.

Área metropolitana de Barcelona, 2001

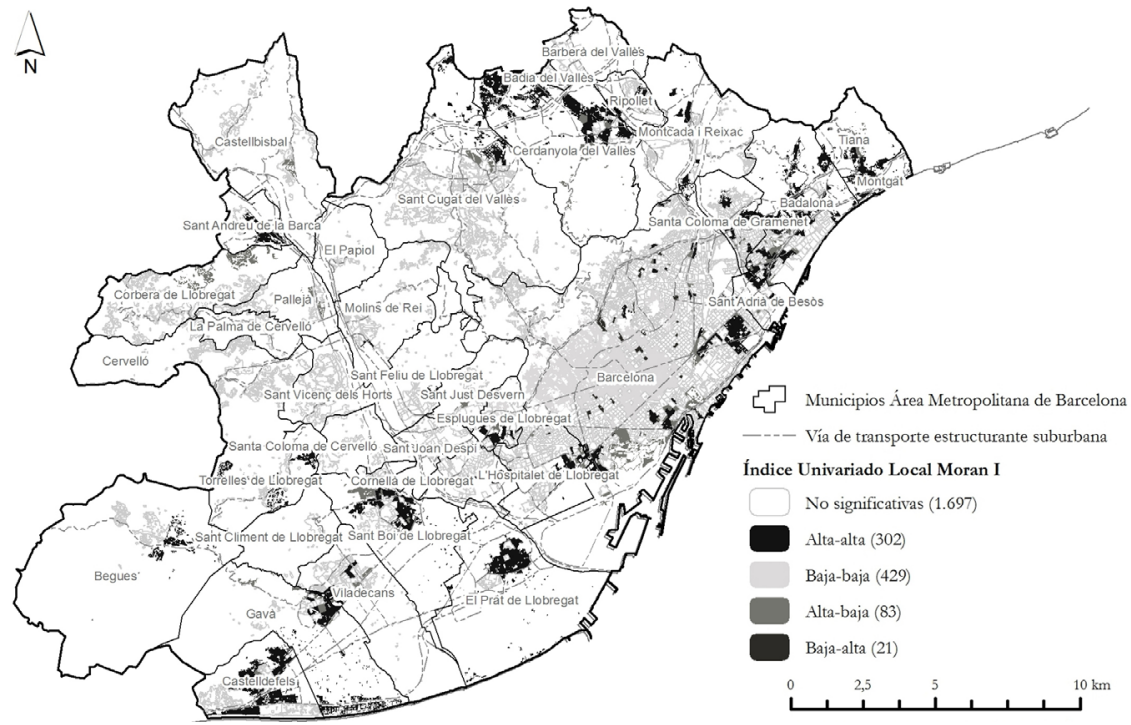

FUENTE ELABORACIÓN PROPIA. BASES CARTOGRÁFICAS: INSTITUT CARTOGRÀFIC DE CATALUNYA. DATOS CENSALES: 2OOI, INSTITUTO NACIONAL DE ESTADÍSTICAS, GOBIERNO DE ESPAÑA 
FIgURA I I I Índice Local de Moran (LISA) sobre la categoría ocupacional de trabajadores semicualificados de servicios. Área metropolitana de Barcelona, 2011

$\widehat{N}_{\mathrm{N}}$

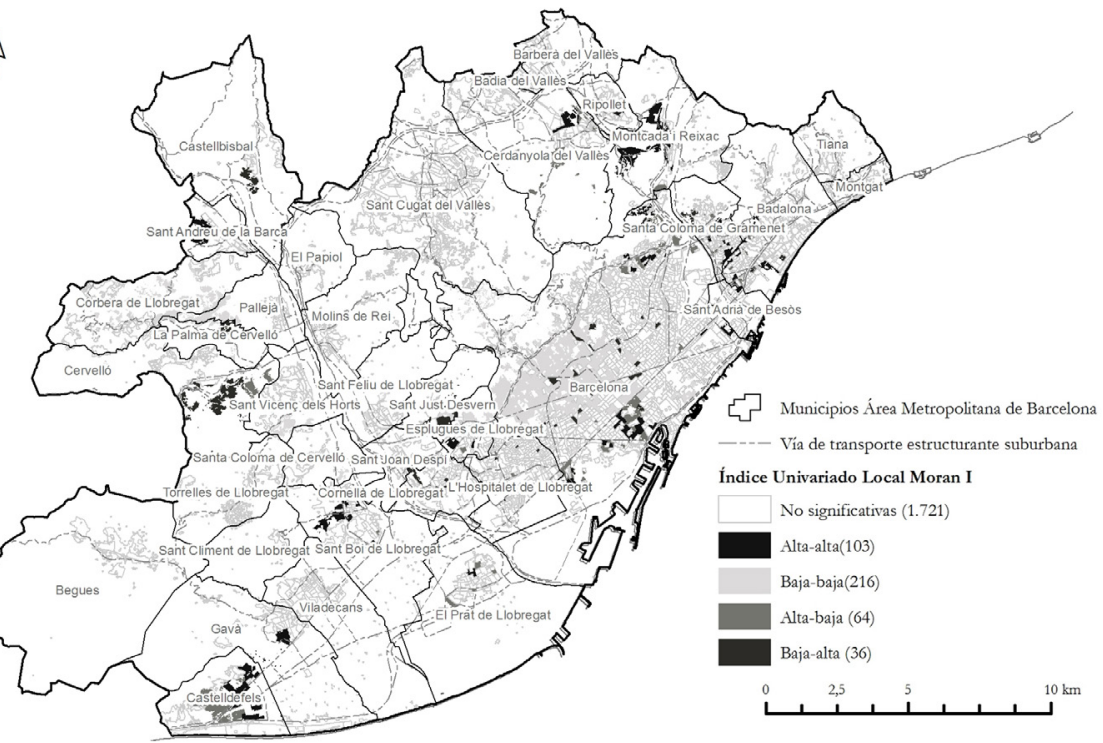

FUENTE ELABORACIÓN PROPIA. BASES CARTOGRÁFICAS: INSTITUT CARTOGRÀFIC DE CATALUNYA. DATOS CENSALES: 2OI I, INSTITUTO NACIONAL DE ESTADÍSTICAS, GOBIERNO DE ESPAÑA

Si se cuantifica la proporción de secciones censales que ocupan cada uno de los grupos estudiados siguiendo un patrón residencial agrupado (alta-alta), se puede observar claramente que los profesionales y directivos ocupan un mayor espacio de la metrópolis de Barcelona en comparación con los trabajadores de servicios semicualificados. Además, esta diferencia ha aumentado durante la primera década de los 2000 de manera significativa, coincidiendo con la segunda etapa de terciarización económica y la llegada de la crisis (figura 12). En este periodo, la concentración residencial de los trabajadores semicualificados de servicios ha perdido claramente peso en la estructura residencial barcelonesa, situándose incluso en valores inferiores a los que presentaba a principios de los años noventa. Con estos resultados, todo indica que es justamente en el periodo en que aumenta más la desigualdad social cuando la separación residencial entre los grupos que polarizan la estructura ocupacional pierde intensidad, particularmente en el caso de los trabajadores semicualificados de servicios. Mientras que la clase de servicio (profesionales y directivos) va ocupando de forma compacta cada vez más áreas de la ciudad central, particularmente zonas céntricas y regeneradas, la nueva clase trabajadora (trabajadores semicualificados de servicios) ha ido abandonando las zonas céntricas, expandiéndose por la metrópoli sin seguir un patrón de localización residencial claro, solo agrupándose de forma significativa en la periferia. 
FIGURA I2 | Proporción de secciones censales identificadas como "alto-alto" por el Î́ndice Local de Moran según categoría ocupacional. Área metropolitana de Barcelona, 1991-2011 (\%)

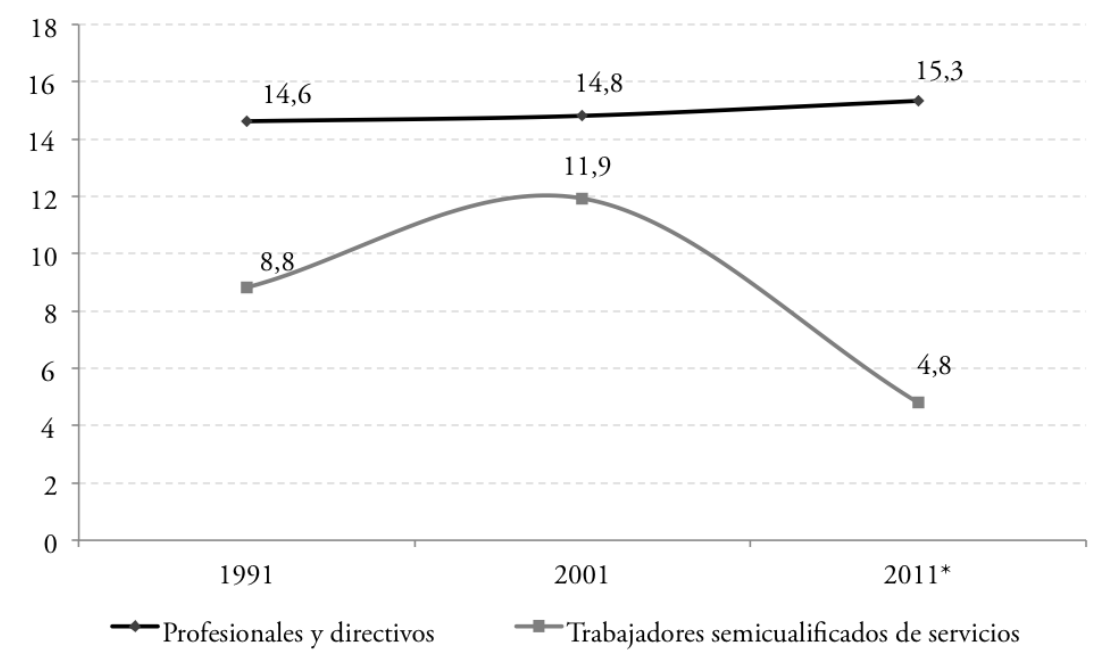

FUENTE INE. CENSO DE POBLACIÓN Y VIVIENDA, I 99 I-2OI I

* DATOS FALTANTES imputados POR El IERMB A PARTIR DEL SOFTWARE IVEWARE

Hay diversos factores que se pueden asociar a este comportamiento residencial. Por un lado, en el caso de la clase de servicio, hay que tener en cuenta los intensos procesos de regeneración urbana que se han llevado a cabo durante las últimas décadas en Barcelona, especialmente en Ciutat Vella (Arbaci \& Tapada-Bertelli, 2012) y en el Poblenou, a partir del Plan 22@ (Ajuntament de Barcelona, 2011), los cuales han habilitado nuevas zonas residenciales para clases medias en la ciudad. A ello hay que sumar la transformación del litoral barcelonés, con efectos en la misma línea. Por otro lado, en el caso de los trabajadores semicualificados de servicios, hay que tener presente que la segunda fase del último boom inmobiliario español (1997-2007) estuvo marcada por la apertura del acceso a créditos hipotecarios a amplios sectores de la sociedad, una circunstancia que aumentó significativamente la movilidad residencial de la clase trabajadora. De hecho, la llegada incesante de población extranjera con el cambio de siglo también influyó en este comportamiento residencial de la clase trabajadora autóctona, ya que permitió la venta de antiguos inmuebles a la población extranjera, de manera que la clase trabajadora autóctona comenzó a adquirir capital para cambiar de residencia a zonas de nueva construcción (Módenas, 2007). Precisamente esto ha contribuido a que la población inmigrante haya seguido un patrón de asentamiento residencial disperso e integrado con la población autóctona, ubicándose principalmente en cascos antiguos y barriadas obreras (Musterd \& Fullaondo, 2008). Es esto lo que explicaría que, a pesar de la intensa llegada de inmigración internacional, no se hayan conformado claramente enclaves étnicos en el área metropolitana de Barcelona (Galeano, Sabater \& Domingo, 2014), los cuales podrían haber hecho aumentar la segregación residencial socioeconómica. 


\section{Conclusiones}

Los análisis realizados a lo largo de este artículo ponen de manifiesto que la evolución de la metrópoli de Barcelona no se ajusta a los parámetros de ciudad dual planteados por Saskia Sassen (1991). Además, los resultados ponen en valor muchas de las críticas dirigidas a la universalidad de este modelo teórico, argumentadas principalmente por autores europeos. A lo largo del análisis se ha observado cómo, efectivamente, los efectos redistributivos del Estado de Bienestar (Marcuse \& Van Kempen, 2000; Van der Wust \& Musterd, 1998; Van Kempen \& Murie, 2009) y de la estructura de los hogares (Hamnett, 1994), así como las particularidades contextuales de las dinámicas de accesibilidad a la vivienda (Maloutas, 2012), pueden incidir de forma relevante en la estructuración socioespacial de las grandes ciudades, más allá de la influencia que se deriva de los procesos de desindustrialización y de globalización económica.

Durante el periodo 1991-2001, a pesar de la culminación de la primera etapa de terciarización del sistema productivo, la desigualdad social no aumentó en la conurbación barcelonesa, sino todo lo contrario. La combinación de factores como el desarrollo del Estado del Bienestar durante las primeras legislaturas democráticas, el aumento incesante del número de ocupados durante todo el periodo y también la intensa incorporación de la mujer al mercado de trabajo a finales de la década, situaron a la sociedad metropolitana en un largo proceso de cohesión social, siguiendo un modelo de crecimiento inclusivo (Trullén, 2015) que precisamente se opone al modelo de ciudad dual. En cuanto a la dinámica de segregación residencial socioeconómica, tampoco se incrementó durante este periodo, manteniéndose a un nivel moderado.

El periodo 2001-2011, en cambio, está claramente marcado por el impacto de la crisis económica, el cual intensificó la segunda etapa de terciarización económica. La estructura ocupacional terminó de polarizarse y la desigualdad social aumentó por primera vez en las dos últimas décadas, pero se trata de dos fenómenos que tienen más que ver con la destrucción de empleo ocasionada por la crisis, que con la reestructuración del sistema productivo. En todo caso, esta polarización social no se ha traducido de forma clara en un aumento de la segregación residencial socioeconómica en el área metropolitana de Barcelona, sino más bien al contrario. En este sentido, se puede decir que actualmente la metrópoli de Barcelona refleja una perfil de ciudad "desigual-no segregada", utilizando los términos de Fujita (2012), y no de ciudad "desigual-segregada" como correspondería a una ciudad dual. 


\section{Referencias bibliográficas}

Ajuntament de Barcelona (2011). 22@Barcelona: 10 anys de renovació urbana. Barcelona: Ajuntament de Barcelona.

Anselin, L. (1995). Local Indicators of Spatial Association-LISA. Geographical Analysis, 27(2), 93-115. http://dx.doi.10.1111/j.1538-4632.1995.tb00338.x

Anselin, L., Syabri, I. \& Kho, Y. (2006). GeoDa: An introduction to spatial data analysis. Geographical Analysis, (38), 5-22. http://dx.doi.10.1111/j.0016-7363.2005.00671.x

Arbaci, S. \& Tapada-Bertelli, T. (2012). Social inequality and urban regeneration in Barcelona city center: reconsidering success. European Urban and Regional Studies, 19(3), 287-311.

Atkinson, R. \& Kintrea, K. (2001). Disentangling area effects: Evidence from deprived and non-deprived neighbourhoods. Urban Studies, 38(12), 2277-2298. http://dx.d oi.10.1080/00420980120087162

Bell, D. (1976). El advenimiento de la sociedad post-industrial: un intento de prognosis social. Madrid: Alianza.

Bernardi, F., Garrido, L. \& Miyar, M. (2011). The recent fast upsurge of immigrants in Spain and their employment patterns and occupational attainment. International Migration, 49(1), 148-187. http://dx.doi.10.1111/j.1468-2435.2010.00610.x

Burgers, J. \& Musterd, S. (2002). Understanding urban inequality: A model based on existing theories and an empirical illustration. International Journal Of Urban And Regional Research, 26(2), 403-413. http://dx.doi.10.1111/1468-2427.00387

Castells, M. (1995). La ciudad informacional: tecnologías de la información, reestructuración económica y el proceso urbano-regional. Madrid: Alianza Editorial.

Castells, M. \& Mollenkopf, J. (1991). Conclusion: Is New York a dual city. En J. Mollenkopf \& M. Castells, Dual City: Restructuring New York (pp. 399-418). Nueva York: The Russell Sage Foundation.

Duncan, O. \& Duncan, B. (1955a). A methodological analysis of segregation indexes. American Sociological Review, 20(2), 210-217. http://dx.doi.10.2307/2088328

Duncan, O. \& Duncan, B. (1955b). Residential distribution and occupational stratification. American Journal of Sociology, 60(5), 493-503.

Esping-Andersen, G. (Ed.). (2002). Why we need a new Welfare State. Nueva York: Oxford University Press.

Fernández-Macías, E., Hurley, J. \& Storrie, D. (2012). Transformation of the employment structure in the eu and USA, 1995-2007. Basingstoke, UK: Palgrave Macmillan.

Fujita, K. (2012). Conclusion: Residential segregation and urban theory. En T. Maloutas \& K. Fujita, Residential segregation in comparative perspective: Making sense of contextual diversity (pp. 285-322). Surrey: Ashgate Publishing.

Fullaondo, A. (2007). La inmigración en España: una aproximación metropolitana comparada. Arquitectura, Ciudad y Entorno, 2(4), 497-518. En https://dialnet.unirioja.es/ ejemplar/240405

Galeano, J. M., Sabater, A. \& Domingo, A. (2014). Formació i evolució dels enclavaments ètnics a Catalunya abans i durant la crisi econòmica. Documents d'Anàlisi Geogràfica, 6O(2), 261-288. http://dx.doi.org/10.5565/rev/dag.111 
Gottschalck, P. \& Smeeding, T. M. (1997). Cross-national comparisons of earnings and income inequality. Journal Of Economic Literature, (35), 633-687. En http://darp.lse.ac.uk/ PapersDB/Gottschalk-Smeeding_(JEL97).pdf

Gregg, P. y Wandsworth, J. (2002). Why we should (also) Measure Worklessness at the Household Level. Theory and evidence from Britain, Spain, Germany and the United States. Department of Economics, University of Bristol, Working Papers Series, (02/053).

Hamnett, C. (1994). Social polarisation in global cities: Theory and evidence. Urban Studies, 31(3), 401-424. http://dx.doi.10.1080/00420989420080401

Hamnett, C. (1998). Social polarisation, economic restructuring and Welfare State regimes. En S. Musterd \& W. Ostendorf, Urban segregation and the Welfare State: Inequality and exclusion in western cities (pp. 15-27). Londres: Routledge.

Harloe, M. \& Fainstein, S. S. (1992). Conclusion: The divided cities. En S. S. Fainstein, I. Gordon \& M. Harloe, Divided Cities: New York \& London in the Contemporary World (pp. 236-268). Oxford: Blackwell.

Laparra, M. \& Pérez, B. (2012). Crisis y fractura social en Europa. Causas y efectos en España. Barcelona: La Caixa.

Lesthaeghe, R. (1995). The second demographic transition in Westerns countries. En K. Oppenheim \& A. Jensen, Gender and family change in industrialized countries (pp. 17-62). Oxford: Clarendon Press.

Maloutas, T. (2012). Introdution: Residential segregation in context. En T. Maloutas \& K. Fujita, Residential segregation in comparative perspective: Making sense of contextual diversity (pp. 1-36). Surrey: Ashgate publishing.

Marcuse, P. (1989). 'Dual city': A muddy metaphor for a quartered city. International Journal of Urban and Regional Research, 13(4), 697-708. http:// dx.doi.10.1111/j.1468-2427.1989.tb00142.x

Marcuse, P. (1993). What's so new about divided cities. International Journal of Urban and Regional Research, 17(3), 355-365. http://dx.doi.10.1111/j.1468-2427.1993.tb00226.x

Marcuse, P. (1996). Space and race in the post-fordist City: The outcast ghetto and avanced homelessness in the United States Today. En E. Mingione, Urban poverty and the underclass: A reader (pp. 176-216). Oxford: Blackwell.

Marcuse, P. \& Van Kempen, R. (2000). Conclusion: A changed spatial order. En P. Marcuse \& R. Van Kempen, Globalizing Cities: A New Spatial Order? Oxford: Blackwell.

Martín, A., López-Roldán, P. \& Molina, Ó. (2011). Movilidad ascendente de la inmigración en España: ‘asimilación o segmentación ocupacional. Papers, 96(4), 1.335-1.362. En http://papers.uab.cat/article/view/v96-n4-lopez-molina-martin/papers_96_4-martin_ artiles

Massey, D. S. \& Denton, N. A. (1988). The dimensions of residential segregation. Social Forces, 67(2), 281-315. http://dx.doi.10.2307/2579183

Massey, D. S. \& Denton, N. A. (1993). American Apartheid: Segregation and the making of the underclass. Cambridge, ma: Harvard University Press.

Méndez, R. \& Caravaca, I. (1993). Procesos de reestructuración industrial en las aglomeraciones metropolitanas españolas. Madrid: Ministerio de Obras Públicas y Transporte (морт).

Méndez, R. \& Prada-Trigo, J. (2014). Crisis, desempleo y vulnerabilidad en Madrid. Scripta Nova. Revista Electrónica de Geografía y Ciencias Sociales, 18(47), 1-13. En http://www. ub.edu/geocrit/sn/sn-474.htm 
Mingione, E. (1996a). Conclusion. En E. Mingione, Urban poverty and the underclass: A reader (pp. 370-383). Oxford: Blackwell.

Mingione, E. (1996b). Urban poverty in the advanced industrial world: Concepts, analysis and debates. En E. Mingione, Urban poverty and the underclass: A reader (pp. 3-40). Oxford: Blackwell.

Módenas, J. A. (2007). Una visión demográfica de la movilidad residencial reciente en España. En J. M. Feria (Ed.), La vivienda y el espacio residencial en las áreas metropolitanas. Sevilla: Centro de Estudios Andaluces.

Moreno, L. \& Sarasa, S. (1992). Génesis y desarrollo del Estado del Bienestar en España. Instituto de Estudios Sociales Avanzados. Documento de Trabajo, (92-13). En https:// dialnet.unirioja.es/servlet/articulo?codigo $=1367666$

Musterd, S. \& Fullaondo, A. (2008). Ethnic segregation and the housing market in two cities in northern and southern Europe: The cases of Amsterdam and Barcelona. Arquitectura, Ciudad y Entorno, 3(8), 93-114. En http://dare.uva.nl/search?metis.record.id=294508

Nel.lo, O. (1994). L'impacte social de la reestructuració industrial a la regió metropolitana de Barcelona. Papers. Regió Metropolitana de Barcelona, (18), 65-81. En http://www.raco. cat/index.php/PapersIERMB/article/view/101334

Pierson, P. (1998). Irresistible forces, inmovable objects: post-industrial welfare states confront permanent austerity. Journal of European Public Policy, 5(4), 539-560.

Préteceille, E. (1995). Division sociale de l'espace et globalisation: le cas de la métropole parisienne. Sociétés Contemporaines, (22/23). http://dx.doi.10.3406/socco.1995.1530

Raghunathan, T. E., Lepkowski, J. M., Van Hoewyk, J. \& Solenberger, P. (2001). A multivariate technique for multiply imputing missing values using a sequence of regression models. Survey Methodology, 27(1), 85-95. En http://bit.ly/2r2bHMd

Reichl, A. (2007). Rethinking the dual city. Urban Affairs Review, 42(5), 659-687. http:// dx.doi.10.1177/1078087406298118

Rodríguez, J. (2006). Los booms inmobiliarios en España: un análisis de tres periodos. Papeles de Economía Española, (109), 76-90.

Sarasa, S., Porcel, S. \& Navarro-Varas, L. (2013). L'impacte social de la crisi a l’Àrea Metropolitana de Barcelona i a Catalunya. Papers. Regió Metropolitana de Barcelona, (56), 10-88. En http://www.raco.cat/index.php/PapersIERMB/article/view/265059

Sassen, S. (1991). The global City: New York, London, Tokyo. Princeton, NJ: Princeton University Press.

Sassen, S. (1994). Cities in a world economy. Thousand Oaks, ca: Pine Forge Press.

Sassen, S. (1996). Service employment regimes and the new inequality. En E. Mingione, Urban poverty and the underclass: A reader (pp. 64-82). Oxford: Blackwell.

Swank, D. (2002). Global capital, political institutions, and policy change in developed welfare states. Cambridge: Cambridge University Press.

Towsend, P. (1993). The international analysis of poverty. NuevaYork: Routledge.

Trullén, J. (1998). El modelo Barcelona de desarrollo económico-urbanístico: a la búsqueda de flexibilidad territorial. En Q. Brugué \& R. Gomà, Gobiernos locales y políticas públicas. Bienestar social, promoción económica y territorio. Barcelona: Ariel.

Trullén, J. (2001). El territori de Barcelona cap a l'economia del coneixement. Barcelona: Diputación de Barcelona. 
Trullén, J., Lladós, J. \& Boix, R (2002). Economía del conocimiento, ciudad y competitividad. Investigaciones Regionales, (1), 139-161. En https:/dialnet.unirioja.es/descarga/ articulo/2124408.pdf

Uyeki, E. S. (1964). Residential distribution and stratification, 1950-1960. American Journal Of Sociology, 69(5), 491-498. http://dx.doi.10.1086/223652

Van Kempen, E. (1994). The dual city and the poor: Social polarisation, social segregation and life chances. Urban Studies, 31(7), 995-1015. http://dx.doi.10.1080/00420989420080911

Van Kempen, R. \& Murie, A. (2009). The new divided city: Changing patterns in European cities. Tijdschrift Voor Economische en Sociale Geografie, 100(4), 377-398. http:// dx.doi.10.1111/j.1467-9663.2009.00548.x

Van Weesep, J. \& Van Kempen, R. (1992). Economic change, income differentiation and housing: urban response in the Netherlands. Urban Studies, 29(6), 979-990.

Van der Wusten, H. \& Musterd, S. (1998). Welfare State effects on inequality and segregation: Concluding remarks. En S. Musterd \& W. Ostendorf, Urban segregation and the Welfare State: Inequality and exclusion in western cities (pp. 238-247). Londres: Routledge.

Wacquant, L. (2001). Parias urbanos: marginalidad en la ciudad a comienzos del milenio. Buenos Aires: Manantial.

Wacquant, L. (2007). La estigmatización territorial en la edad de la marginalidad avanzada. Ciências Sociais Unisinos, 43(3), 193-199. En http://www.redalyc.org/ pdf/938/93843301.pdf

White, P. (1984). The West European City: A social geography. Essex: Longman.

Wilson, W. J. (1987). The truly disavantaged: The inner city, the underclass, and public policy. Chicago: The University Chicago Press. 